\title{
Influencia asiática en la producción de mezcal en la costa de Jalisco. El caso de la raicilla
}

DOI: $10.32870 /$ mycp.v11i33.317

\author{
Ana G. Valenzuela Zapata* \\ Aristarco Regalado Pinedo \\ Michiko Mizoguchi
}

\begin{abstract}
Resumen
Probablemente en el siglo XIII ya se elaboraban destilados de arroz en Tailandia con un aparato destilador rústico que llegó a las zonas templadas de Asia y que derivó en las bebidas conocidas como shochu en el sur de Japón y mas tarde en el siglo XVI en la producción de un destilado de agave o mezcal en la costa de Jalisco. Con los nombres de shochu y de mezcal se distinguen en Japón y en México una gran diversidad de aguardientes; en el primero, procedente de diversas materias primas, y en el segundo principalmente de especies y variedades de agaves. Sin embargo, algunos shochu de las islas de Kuyshu y Okinawa, así como algunos mezcales como la raicilla de El Tuito (municipio de Cabo Corrientes) y los mezcales de Zapotitlán de Vadillo y el de olla de la Manzanilla de la Paz, comparten una misma técnica conocida en México como destilador huichol o asiático y en Japón como "kabutogama chiki”. El conocimiento de la destilación llegó a México después de la conquista y se dispersó no necesariamente por una sola vía, como lo sugieren algunos autores a partir del modelo del "vino de cocos" en el caso del aparato asiático hasta llegar a la tradición tequilera. Tampoco obligatoriamente debieron tener un origen asiático quienes sabían hacer funcionar las técnicas de destilación asiáticas y que lograron sustituir los materiales en cada región a lo largo de la costa occidental de México. De una simplicidad mayor a la de la tradición de los alambiques árabes, esta técnica sobrevive y enlaza una historia entre el Pacifico asiático y el mexicano, que ha sido poco estudiada y que después de 800 años es útil y sobrevive en las comunidades con bebidas microrregionales en ambos continentes.
\end{abstract}

Palabras clave: mezcal, raicilla, shochu, destilación asiática

* Profesora investigadora de la Universidad de Guadalajara en el Centro Universitario de la Ciénega, Departamento de Negocios.

** Profesor de la Universidad de Guadalajara en el Centro Universitario de Ciencias Sociales y Humanidades, Departamento de Estudios del Pacífico.

*** Periodista y traductora. Coordinadora del programa "Explorando el patrimonio cultural de la humanidad. Paisaje agavero en la región de Tequila”, NHK (televisión pública japonesa). 
Ana G. Valenzuela Zapata, Aristarco Regalado Pinedo y Michiko Mizoguchi

Abstract

Perhaps in XIII century in Thailand some rice ferments were distilled with a simple still technique influencing also northern Asia countries as China, Korea and specially in southern Japan an spirit named as shochu, after that in XVI century this equipment was used also in a Mexican distilled production with agave plants named mescal in Jalisco coastal areas. Shochu and mescal are spirits in Japan and Mexico used to talk about a big diversity of alcoholic beverages, in the first one raw materials have a wide origin (seeds, fruits, potatoes, etc) and in the second one just agave species and varieties are cooked for. Some shochu spirits from Kuyshu and Okinawa islands and some "mezcales" named raicilla from El Tuito (Cabo Corrientes municipality) and others from Zapotitlán de Vadillo and "mescal de olla" from Manzanilla de la Paz with huichol or Asiatic still and named in Japan as "kabutogama chiki". Distillation was known in Mexico after conquest and their spread knowledge was not strictly conducted by a one region or way in geography and coconut wine pattern as some authors are suggested and after developed in tequila tradition. Also, asiatics or not were making the equipment of hucihol or Asiatic still in different versions and materials along pacific coast. Being a simple technique Asiatic still than Arabian alambique still, this is a link between Asian and Mexican pacific coast, few studies are made and after eight centuries ago people is working with that and producing traditional beverages in micro regions.

Key words: agave spirits, mescal, raicilla, shochu, Asiatic stills

\section{Alcances del estudio}

A falta de información sobre el destilado raicilla y desde una perspectiva interdisciplinaria entre la historia y la botánica económica, en este trabajo de investigación indagamos sobre los conocimientos tradicionales del uso de agaves y destiladores. Hacemos un especial acercamiento a la destilación por tratarse de un tema en discusión arqueológica, que merece abrir el interés de grupos de estudio interdisciplinario para validar las hipótesis del origen de la destilación y la influencia asiática en la costa del Pacífico mexicano. Utilizamos la comparativa con un destilado japonés, debido a la cantidad de información sistematizada que existe para el shochu, no así para otras bebidas asiáticas que han transformado sus tecnologías sin dejar muestra de los ancestrales aparatos de destilación, como el kabutogama chiki, del cual hablaremos más adelante. Otra de las justificaciones para contribuir al conocimiento de la raicilla es el interés de los productores por acrecentar la información de la cultura de la bebida y proponer nuevas certificaciones al producto ligadas a su antigüedad, historia, tipicidad y patrimonio. 


\section{La destilación y sus técnicas}

La destilación ${ }^{1}$ de perfumes y alcoholes es atribuida a los árabes, pero en Europa la técnica fue usada para destilar vinos y conseguir el remedio aqua vitae, que se transformó en el curso del siglo xv en una bebida "recreacional" (Standage, 2005: 100). Paralelamente durante los siglos XIII y XIV otra técnica con la que se destilaban fermentos de arroz se difundió de Asia tropical a China, Corea y Japón. (http://www.japansake.or.jp/honkaku/about/all/history. html). A grandes rasgos, éstas fueron las dos formas de destilación utilizadas en la época anterior al siglo del primer viaje alrededor del mundo, es decir el siglo XVI. Los dos mecanismos de destilación, el asiático y el árabe, llegaron a tierras mexicanas con la Conquista y colonización española, y con ellos se lograron destilados de fermentos de mezcal. Dichos fermentos se elaboraban de acuerdo a una tradición prehispánica en la que se cocían o tatemaban las "cabezas" o "piñas"2 de diferentes especies del género Agave L, usadas ancestralmente como alimentos. Los aparatos destiladores de influencia árabe dominan en la actualidad en la industria de los destilados a escala mundial, y México no es una excepción. El destilador asiático, también llamado "huichol" por Lummholtz, es menos conocido (Bruman, 1940; Walton, 1977, citados por Illsey, 2005), sobrevive en diversas regiones gracias a la producción de pequeños volúmenes de alcoholes o bebidas destiladas en México y Asia.

Serra et al. (2005) muestran en sus estudios la gran diversidad de mezcales producidos con ambas tecnologías ("Etnoarqueología del mezcal"). Algunos destilados de shochu y raicilla, producidos en el sur de Japón y en la costa de Jalisco, respectivamente, el primero con materias primas diversas y el segundo principalmente con agaves, mantienen el uso del aparato asiático para destilar. Mientras que en el shochu existen ciertos estudios del aparato llamado kabutogama chiki, en Jalisco se inician las investigaciones de la influencia asiática en la tradición mezcalera (Colunga y Zizumbo, 2006: 1-15).

Una de las formas más conocidas de mezcal en México es el tequila, inicialmente llamado "vino mezcal de tequila", originario de Jalisco y ahora famoso

1. Separar por medio del calor, en alambiques u otros vasos, una sustancia volátil de otras más fijas, enfriando luego su vapor para reducirla nuevamente a líquido (Real Academia Española. Disponible en: http://www.rae.es/).

2. Con el nombre de "cabeza" o "piña" se denomina la forma esférica del tallo y las vainas de las hojas de agaves que conservan polisacáridos antes de la floración y que con la cocción se transforman en azúcares fermentescibles. 
por el mundo y legitimado como una denominación de origen, un paisaje natural y cultural en el registro UNESCO y una producción de 280 millones de litros en 2007 (Macías y Valenzuela, 2007: 1-2). El tequila es descendiente de la tradición mezcalera arraigada en el occidente de México, en Jalisco, cuyas bases están cimentadas en esa herencia cultural que comparte con otros mezcales que no han llegado a tener una denominación de Origen, pero que actualmente son inventariadas por la Comisión Nacional de la Biodiversidad (Conabio) para registrar los recursos y sus patrimonios naturales y culturales y promover su conservación (Eguiarte, 2003; Conabio, 2005).

Con el objetivo de contribuir al conocimiento de la producción de mezcales y raicillas de Jalisco y su influencia asiática, realizamos esta investigación a partir de una revisión histórica y un trabajo de campo que comprende entrevistas no estructuradas en visitas a tabernas de producción familiar artesanal de noviembre 2007 a noviembre 2008 en los municipios de Cabo Corrientes, Tonaya, Chiquilistlán, Mazamitla, Concepción de Buenos Aires, Mascota y San Sebastián del Oeste, y Zapotitlán de Vadillo en junio de 2001. La identificación de especies vivas en plantación o en recolecta de agaves, se basó en la obra de Gentry (1982). De la investigación del proceso artesanal para la producción de mezcal, se interrogó a los productores sobre todo acerca del aparato destilador utilizado, y a los distribuidores y consumidores sobre la calidad de los productos. Se revisaron las fuentes bibliográficas, botánicas y de producción de la bebida shochu, se entrevistaron funcionarios de las oficinas independientes del National Research Institute of Brewing en Japón. Finalmente, también hicimos trabajo de archivos históricos.

Los resultados se presentan en el primer apartado con la clasificación de los destilados de agave o mezcales, particularmente los de Jalisco y en especial el caso de las raicillas. Proponemos una clasificación de estos destilados con base en sus regiones, especies de agaves usados como materias primas, procesos y características organolépticas. Además de su clasificación, hacemos notar la influencia asiática en su proceso de destilación. En un segundo momento abordamos el caso del shochu de origen japonés, con el fin de demostrar la similitud que existe entre su proceso de destilación con el de algunas raicillas, para abrir el debate y proponer hipótesis sobre la llegada del proceso de destilación asiático a tierras mexicanas. De esta forma, cuestionamos algunas teorías que sostienen que hubo una sola vía de acceso a partir de la cual inició la producción del vino mezcal en Tequila desde donde se dispersó por todo México. 


\section{Destilados de agave}

Los destilados son el producto de la separación de los alcoholes de un fermento de cualquier origen vegetal, sean frutas - como las uvas en el caso del coñac y armagnac-, tubérculos — como la papa en el caso del vodka-, semillas - de cebada para el whisky escocés - o tallos — como la caña de azúcar para el ron-. Algunos aguardientes son pasados por una doble destilación con el fin de que alcancen mayor concentración de alcohol. Hay dos tradiciones ancestrales para destilar. Por un lado el proceso árabe con la utilización del alambique, y por el otro, el método de destilación asiático. Ambas tradiciones dieron lugar a la concepción y fabricación de sendos aparatos. Los árabes produjeron el alambique con serpentín, y los orientales, una torre de destilación sin serpentín, que en este texto llamaremos destilador asiático y que incluye los más de seis modelos que se han fabricado y que han adquirido sus particularidades de acuerdo a cada región geográfica. En efecto, mientras que el destilador asiático se difundió a lo largo de Asia oriental (islas y continente), el alambique invadió el continente europeo durante la época que corresponde a la Edad Media.

Todavía no se sabe con certeza si en América ya se conocían aparatos de destilación en la época prehispánica o si fueron introducidos por los europeos y/o por los asiáticos. El descubrimiento de hornos precolombinos utilizados para la cocción de agaves y la presencia de agave masticado en cuevas (de la misma época) parecen ser, para algunos estudiosos, pruebas suficientes para asegurar que los naturales americanos ya destilaban sus fermentos de agave antes de la llegada de los europeos (Serra et al., 2005). A nosotros nos parece que esas evidencias todavía no constituyen pruebas suficientes para alinearnos con esa posición. Las mencionadas evidencias arqueológicas no hacen más que confirmar lo que ya se sabía gracias a los historiadores o cronistas del siglo XVI. Citemos como ejemplo a fray Toribio de Motolinia. En su obra Historia de los indios de la Nueva España, escrita a mediados del siglo XVI, dedica todo el capítulo 19 para hablar del maguey y de sus usos. En él explica que las pencas y la cabeza del agave se cuecen por separado "y sale de tan buen sabor como un diacitrón no bien adobado o no muy bien hecho". También da una luz respecto al agave masticado: "lo de las pencas está tan lleno de hilos que no se sufre tragarlo, sino mascar y chupar aquel zumo que es dulce..." (Motolinia, 2001: 287). Pero en ningún lugar menciona que los indígenas elaboraran destilados en esa época. 
Por el contrario, lo que sí es seguro es que a principios del siglo XVII el destilado de mezcal ya era muy usado al menos en la costa de la Nueva Galicia. Domingo Lázaro de Arregui es un testigo privilegiado al respecto. No sólo porque vivió y caminó por la Nueva Galicia, sobre todo por la costa, donde tenía su morada (en Tepic), sino porque fue un observador atento. En 1621, tres o cuatro años después de haber incursionado en la sierra de Nayarit para combatir a los tepehuanes, y 13 años antes de su muerte por asma, Arregui escribió una descripción de la Nueva Galicia. El capítulo XXI lo dedicó a "los árboles y plantas de esta tierra". Allí explicó que en la costa ("estas tierras marítimas") utilizaban los mezcales para obtener "un vino por alquitara más claro que el agua y más fuerte que el aguardiente y de aquel gusto" (Arregui, 1980: 106). También agregó que era usado en exceso hasta el grado de haberlo desacreditado, teniendo tantas virtudes. El punto central es que a principios del siglo XVII ya se hacían destilados con el mezcal, y para ello utilizaban una alquitara, es decir un destilador tipo árabe, con serpentín. Cabe destacar que la alquitara es un aparato más rudimentario que el alambique. Es necesario subrayar que el proceso de destilación utilizado en tiempos de Arregui —al menos lo que él mismo observó-, era una técnica introducida por los europeos. Ahora bien, si los indígenas hubieran estado familiarizados con la elaboración de destilados desde tiempos precolombinos, con toda certeza hubieran seguido utilizando sus propios métodos de destilación, al menos al inicio de la época colonial. ¿Acaso hubo razones determinantes para cambiar de manera abrupta sus aparatos destiladores por la alquitara? Permítasenos una comparación arriesgada. Algunos raicilleros de El Tuito nunca dejaron de utilizar su rudimentario aparato destilador asiático a través de los siglos (¿tres, cuatro o cinco siglos?) para adoptar el alambique. En la actualidad todavía utilizan el método asiático frente a personas que se han asociado, en efecto, para sacar provecho de un destilador industrial común de grandes dimensiones, parecido al que utilizan los tequileros en Jalisco. Pero el aparato destilador asiático que llegó a la Nueva Galicia durante la época colonial todavía se sigue usando en Cabo Corrientes. Del mismo modo, si los indígenas de hace cuatro o cinco siglos hubieran cambiado sus destiladores prehispánicos por el alambique, ¿no podría haber subsistido alguna reminiscencia de aquéllos?

Es cierto que los arqueólogos han encontrado recipientes para la fermentación y hornos para la cocción de agaves. También algunos códices muestran imágenes que prueban que los indígenas ya habían domesticado el agave (cortar quiote, dejar madurar la planta durante siete años o más, y 
jimarla para obtener la "piña"). Todo ese proceso es necesario para obtener la fermentación, pero nada prueba que los indígenas hubieran dado el paso siguiente: la destilación para obtener aguardiente. El destilador asiático es muy rudimentario y se puede fabricar actualmente con materiales de fácil acceso: madera del árbol bonete, pencas de agave, recipientes de barro... ahora bien, hay un elemento del que no se puede prescindir para completar ese aparato: se trata del enfriador, necesario para convertir en líquido el vapor. Ese elemento debe ser, forzosamente, un metal. Es decir un recipiente de cobre, por ejemplo, con agua fría que se cambia continuamente para que siempre cumpla con la tarea de enfriar. Hasta la fecha no se ha encontrado este tipo de recipiente de metal en las excavaciones arqueológicas para la época prehispánica. Y si no hay enfriador, es muy difícil la obtención de aguardiente. Estos silencios arqueológicos corroboran los silencios que contienen las fuentes históricas y las crónicas emanadas del primer contacto entre españoles e indígenas. Por eso, la prudencia invita a esperar el momento en que haya más evidencias para aceptar la existencia de un método de destilación entre los habitantes prehispánicos.

Por el contrario, existen en los archivos coloniales multitud de testimonios que indican que la utilización de agaves era común a lo largo y ancho del oeste jalisciense desde la era precolombina. Así lo constataron los conquistadores que por primera vez entraron en contacto con los indígenas de esta

Existen en los archivos coloniales multitud de testimonios que indican que la utilización de agaves era común a lo largo y ancho del oeste jalisciense desde la era precolombina zona en los años 1524-1525. Eran enviados de Hernán Cortés, quien les había encomendado hacer una minuciosa relación escrita sobre toda la zona (Romero de Solís, 1996). Quienes la redactaron pusieron especial cuidado en observar los recursos de la tierra, y escribieron que en la región de Autlán, y entre Etzatlán y Ahuacatlán, producían magueyes. Aseguraron que su uso estaba muy extendido entre todos los pueblos indígenas aunque no fueran productores. Por ejemplo, relataron que desde Cihuatlán hasta Tepic los indígenas utilizaban ropa hecha a partir de alguna especie de agave (AGI. JUSTICIA 113, R. 4). El intensivo uso del agave a lo largo de esta región hacía de ella 
un terreno propicio para el surgimiento rápido de destilados a partir de sus fermentos desde el momento en que se utilizara un aparato destilador. Uno de esos fermentos prehispánicos y que en nuestros días todavía se consume en la costa de Jalisco es la lechuguilla. Se trata de una bebida refrescante y fermentada de jugos de "cabezas" de agave en trozos y ligeramente calentados. En la sierra de Manantlán beben una versión de jugos fermentados provenientes de agaves tatemados que se conoce como "jugo de mezcal", además tiene un uso medicinal (Rafaela Justo, 2008, comunicación personal).

\section{Raicilla}

A grandes rasgos, las bebidas obtenidas del agave las podemos dividir en tres apartados. En el primero se ubican las bebidas resultado del jugo natural de la planta, como el aguamiel. En el segundo apartado caben las bebidas fermentadas y en el tercero, los destilados. Las dos últimas bebidas necesitan pasar por un proceso de elaboración en donde interviene la mano del hombre. Los innumerables mezcales, el bacanora y la raicilla son buenos ejemplos de destilados. Y los fermentos están representados con el pulque y la lechuguilla, aunque exista una gran diferencia entre ambos. Porque el pulque tiene su origen en el aguamiel que se obtiene por un proceso de jugos escurridos en la planta. La bebida "lechuguilla", en cambio, se elabora a partir de la cosecha del tallo o piña (las cabezas) antes de dejarlas fermentar. Precisamente, la lechuguilla y la raicilla siguen un proceso similar que guarda una relación sorprendente hasta el momento de la fermentación. Es decir que, la lechuguilla es el fermento previo a la destilación, pero altamente diluido y sin fibras, es una especie de "agua fresca" ligeramente alcohólica. En ese sentido, los lugares tradicionales de fabricación de lechuguilla ofrecen una pista inapreciable para plantear como hipótesis que allí pudo haber sido la cuna del destilado de mezcal para Jalisco.

Los destilados de mezcal obtenidos en la costa norte y en la sierra occidente de Jalisco son llamados "raicilla". En la actualidad se obtiene raicilla en quince municipios de este estado: Cuautla, Atenguillo, Guachinango, Mascota, San Sebastián del Oeste, Mixtlán, Talpa de Allende (a estos municipios se les considera "zona de origen"), Ayutla, Cabo Corrientes, Puerto Vallarta, Tomatlán, Atengo, Chiquilistlán, Juchitlán, Tecolotlán y Tenamaxtlán (a estos últimos se les considera "zona admitida") (Consejo Mexicano Promotor de la Raicilla, 2000). Esta amplitud regional en la que se produce raicilla impidió 
probablemente que el nombre aludiera a un origen geográfico. En cambio, es plausible que se le llame de ese modo debido a un efecto visual que invita a pensar que es a partir de la raíz del agave de donde se obtiene el destilado. Recurramos otra vez al testimonio de 1621 de Domingo Lázaro de Arregui, cuando aseguró que el mencionado "vino" por alquitara se obtenía de la "raíz y asientos de las pencas" del mezcal (Arregui, 1980: 106). Por supuesto que la versión popular que corre por algunos lugares de la costa relativa al nombre de raicilla es más atractiva. Tal versión refiere que un minero de San Sebastián del Oeste y productor de raicilla, con el fin de burlar las prohibiciones virreinales sobre la producción de licores, bautizó a la bebida bajo el nombre vago de raicilla.

En la zona montañosa (Sierra Occidental: San Sebastián del Oeste, Talpa y Mascota) en bosques de pino y encino y sobre todo en claros donde se encontraban coamiles, pastoreo o cortes forestales, se reproducen agaves de hojas anchas que nombran bajo el mote genérico de "lechuguilla". Reproducidos por semillas, las poblaciones silvestres principalmente de Agave maximiliana, A. inaequidens y recientemente la descubierta $A$. valenciana, se entrecruzan gracias a los murciélagos y se dispersan por el aire para germinar en suelos forestales y ser recolectados para la producción de raicilla. Es frecuente el aprovechamiento de sus ejes florales o quiotes, así como los jugos de las pencas tatemadas como remedios para enfermedades degenerativas crónicas y hasta para elaborar lazos, soguillas y morrales. Estos son, precisamente, las especies de agave que se fermentan y se destilan para obtener raicilla (Hernández et al., 2007). En San Sebastián del Oeste se utiliza un equipo de destilación con componentes híbridos, como el horno, también mencionado por Fritz y Bruman (2000). En el mismo orden de ideas, para el aparato destilador se utilizan torres de destilación y calentador como en el caso del asiático, pero se le ha añadido un serpentín y un tanque enfriador que se obtuvo del modelo del alambique estilo árabe. Además, el horno utilizado es tipo panadero y no el cono truncado cubierto de piedras.

La región costa norte, representada por El Tuito, municipio de Cabo Corrientes, está especialmente relacionada con la producción de una raicilla de gran calidad cuya reputación es bien conocida a lo largo del territorio costero. En la figura 1 -elaborada a partir de Valenzuela (2007) —, se observan las regiones y productos de las diferentes especies del género agave en Jalisco: tequilas, mezcales, lechuguillas, pulques y raicillas. La raicilla de El Tuito tiene diferencias mayores con la producida en San Sebastián del Oeste. En primer 
lugar por las especies de agave que utilizan. En el municipio de Cabo Corrientes se pueden apreciar plantíos de agave angustifolia y de A. rhodacantha en pequeñas parcelas de lomeríos con pendiente ligera, en lo que antes podría haber sido cubierto por bosque tropical húmedo y caducifolio. Se trata de cultivos con procedencia de reproducción asexual (rizomas) que forman un complejo de variedades de hoja angosta. De estas especies existen alrededor de cinco o seis variantes que los productores de raicilla nombran chico aguiar, amarillo, amarillito, cenizo, verde... La segunda diferencia reside en el proceso de destilación. Porque el horno y el aparato destilador de raicilla producida en El Tuito es más parecido al de la tradición japonesa del shoshu. Sobre todo el destilador, compuesto por una torre de madera sobre la que hay un enfriador de metal. En cuanto al horno, también es cierto que es muy similar al utilizado en la época prehispánica, a diferencia del de San Sebastián del Oeste.

\section{Figura 1}

Distribución geográfica de bebidas de agave en Jalisco

\section{DISTRIBUCION GEOGRAFICA DE BEBIDAS DE AGAVE EN JALISCO}

Tequilas, mezcales, lechuguillas, pulque y raicillas
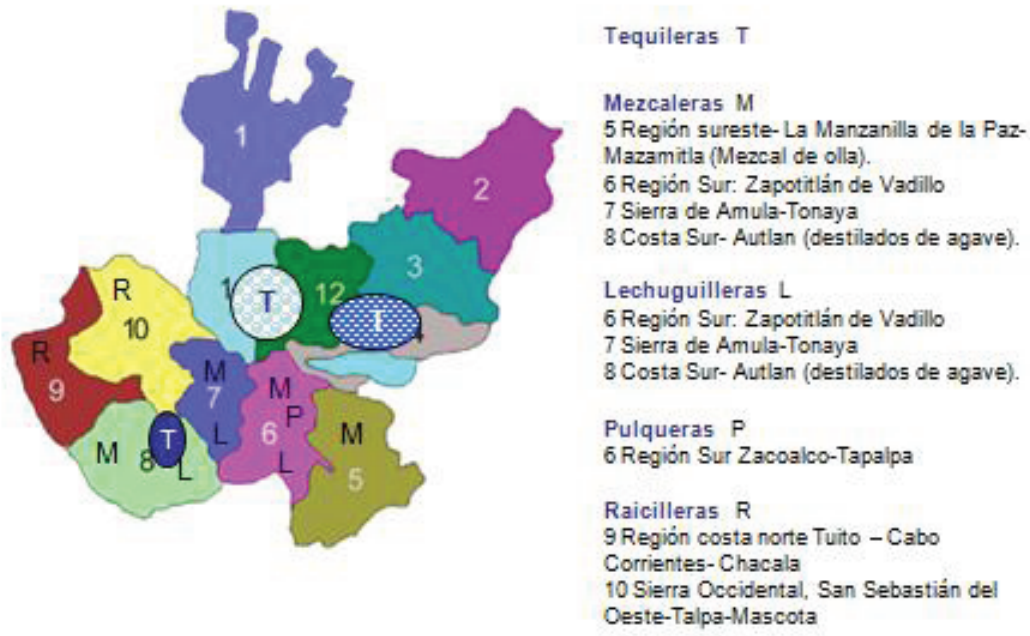

En resumen, se trata de dos regiones que comparten un solo nombre de producto, diferenciándose con el nombre, por el lugar de origen, aquello de mayor reputación por su calidad: raicilla de El Tuito y raicilla de Mascota, 
de la costa norte y sierra occidental del estado de Jalisco, respectivamente. Ambas regiones son limítrofes al núcleo turístico de Puerto Vallarta: al sur se ubica Cabo Corrientes, y al norte y noreste están San Sebastián y Mascota (Jalisco) y Nuevo Vallarta (Nayarit), por lo que también se entiende el auge y la demanda de la raicilla como atractivo. Ligado al turismo, Dachary (2003) propone la ruta de los magueyes de $79 \mathrm{~km}$ cuadrados que incluye además de la región mezcalera, la del tequila: Tonaya y Tuxca de mezcal, Zacolaco de pulque, Autlán con lechuguilla y tequila. La ruta no incluye pulque ni aguamiel de Tapalpa. En el mismo orden de ideas, para el caso de la raicilla, la Dirección de Turismo del municipio de Puerto Vallarta está experimentando con rutas raicilleras, con el objetivo de promover este aguardiente.

Los aparatos destiladores de influencia asiática que encontramos en Jalisco para la producción de mezcal, raicillas y mezcal de olla se presentan en las fotografías de la 1 a la 5 a continuación:

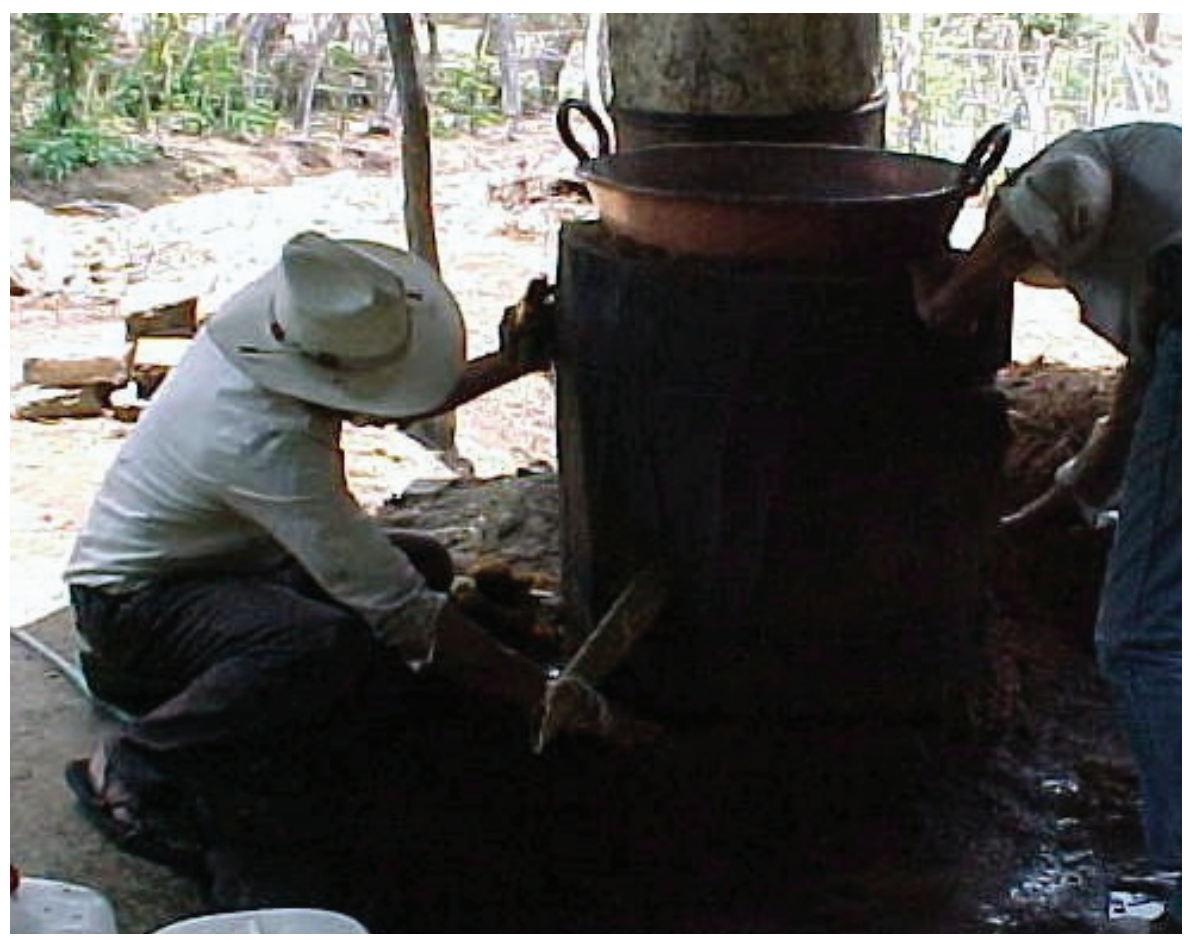

Fotografía 1. Aparato destilador de influencia asiática en Zapotitlán de Vadillo, Jalisco, para elaboración de mezcal. 


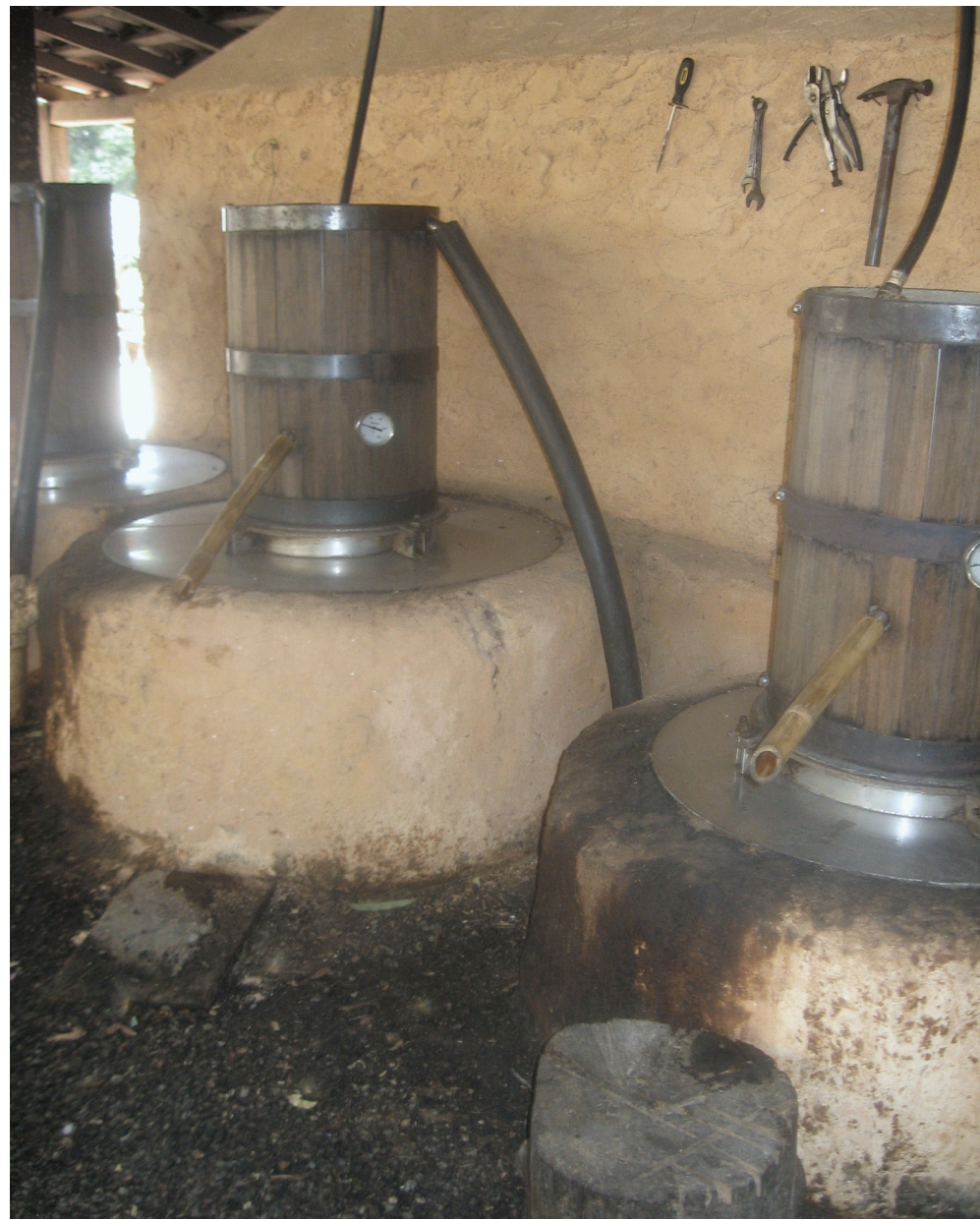

Fotografía 2. Aparato destilador moderno de influencia asiática de El Tuito, municipio de Cabo Corrientes, Jalisco, para elaboración de raicilla. 


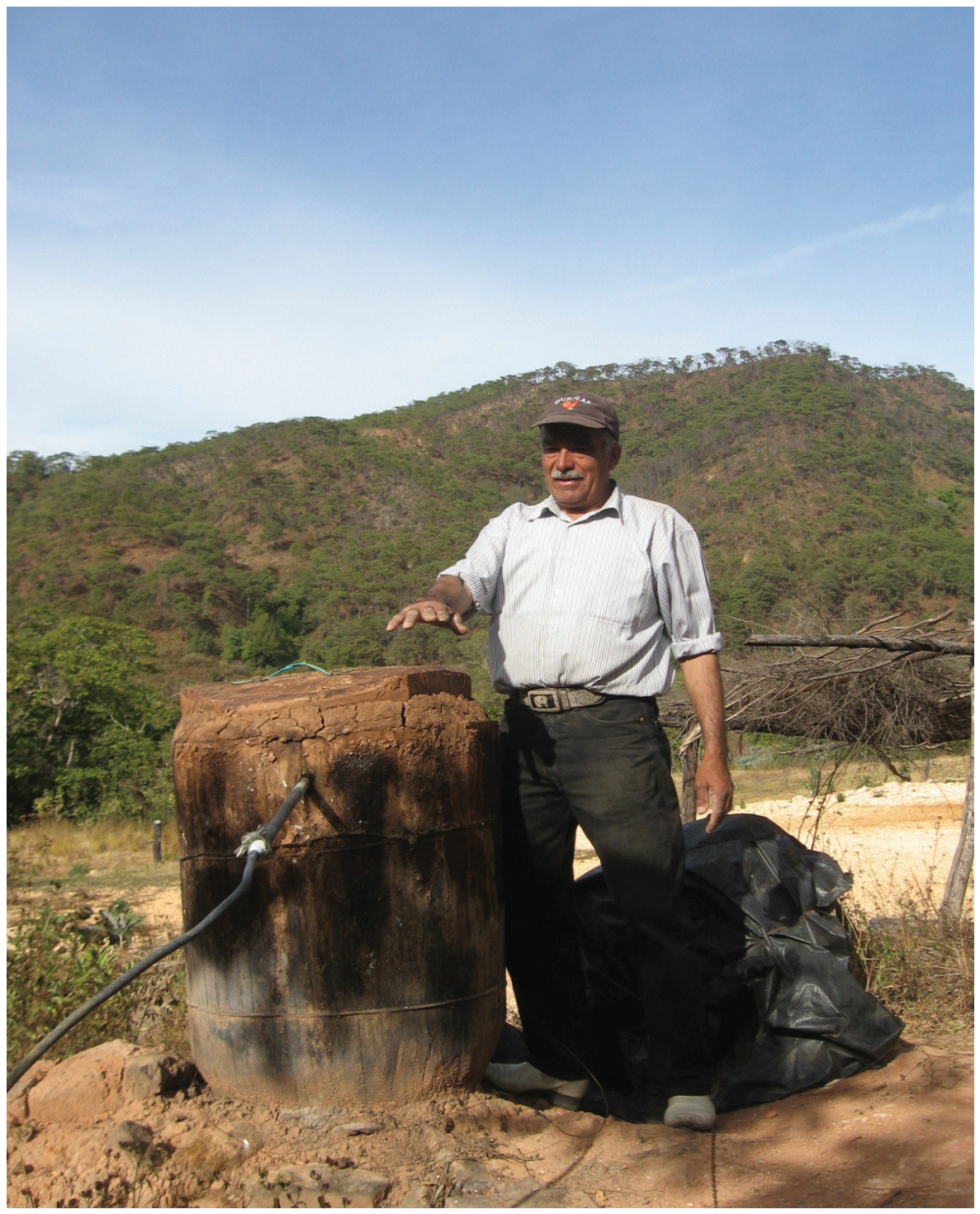

Fotografía 3. Aparato destilador "híbrido" encontrado en San Sebastián del Oeste para elaboración de raicilla. 


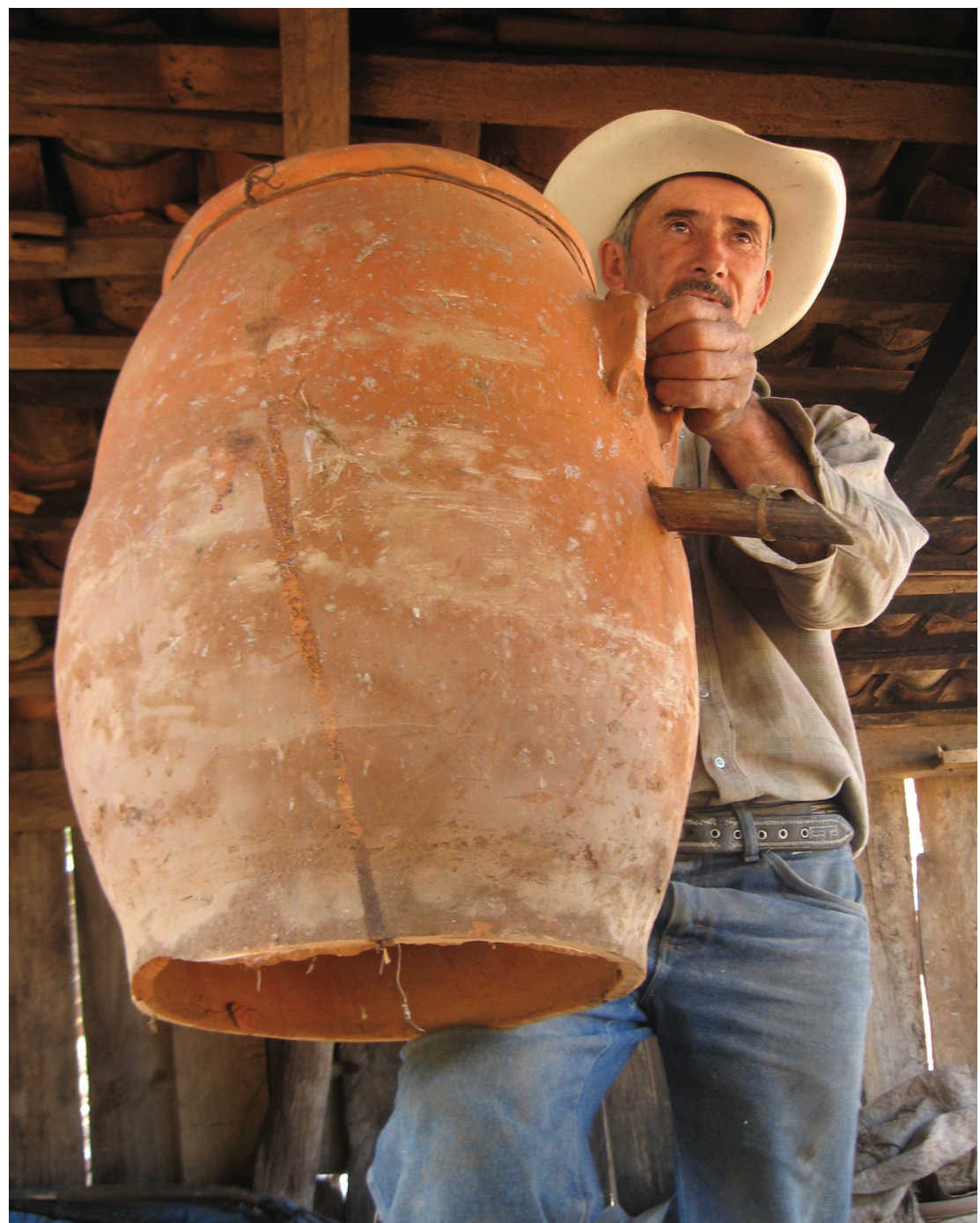

Fotografía 4. Aparato destilador de influencia asiática con sustitución del tronco por la olla, municipio de La Manzanilla de la Paz, para elaboración de "mezcal de olla". 


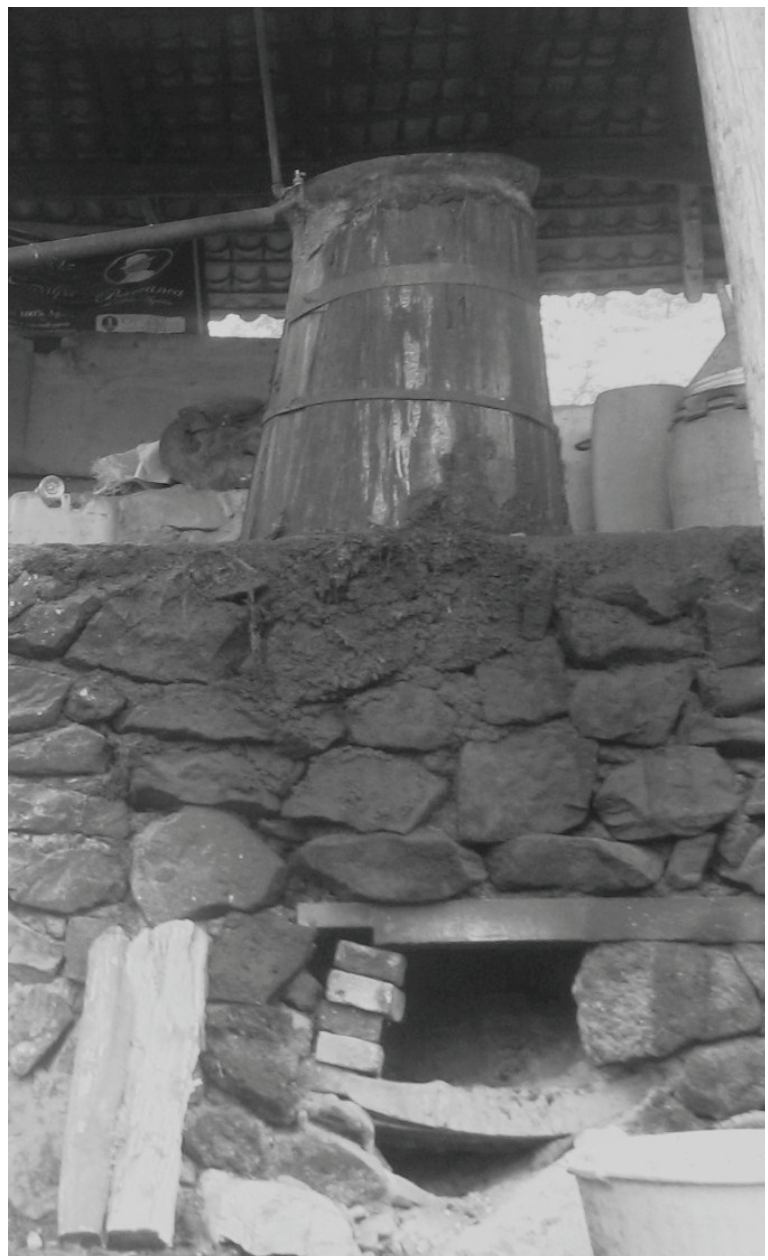

Fotografía 5. Aparato destilador de influencia asiática para elaboración de "barranca". Municipio de Tapalpa.

\section{El shochu, destilado japonés}

La influencia filipina en la producción de mezcal a partir del "vino de cocos" en Colima podría enlazarnos directamente al actual destilado lambanog de la provincia de Infanta (Quezón), que también conserva su sistema tradicional de taberna putuhan (http://www.jcmiras.net/jcm2/p16.htm\#more-16). Sin embargo, decidimos comparar la evolución del aparato destilador kabutogama 
chiki de la tradición japonesa, por contar con mas información histórica que el lambanog. El shochu es una bebida destilada japonesa cuyo nombre significa "concentrado por fuego" y que tiene una sola destilación. Existe una diversidad de materias primas cuyos fermentos se utilizan para hacer este aguardiente: arroz, granos, camote dulce, azúcar y hasta ajonjolí por citar algunas. La diversidad de regiones y de materias primas ha generado una diferenciación de los destilados que en la actualidad retoman auge, en gran medida por su autenticidad. Al igual que otras bebidas étnicas en el proceso de globalización, el shochu se revaloriza culturalmente en Japón. Las bebidas originales han tenido un crecimiento importante y emblemático como bebidas nacionales. Muy especialmente han sido investigados y promovidos comercialmente los shochu del sur del Japón, hechos a base de fermentos de arroz y con una específica levadura llamada koji, también utilizada en la gastronomía japonesa (www.shochu.com).

El origen del shochu se remonta a los siglos XIII ó XIV, cuando ya se producían destilados similares en China o en las islas del mar tropical. Tres rutas pudieron llevar la tecnología de la destilación y del uso de fermentos a Japón. La ruta por Okinawa, cuando en el siglo XIV en Ryukyu (actual prefectura de Okinawa) era la base de comercio marítimo entre China, Corea y Japón. La segunda opción son los piratas (llamados wako) del mar asiático tropical, quienes intercambiaban shochu y licor extranjero, conocido bajo el nombre de nanban shu. Finalmente se ha sugerido que el shochu procede de Tailandia y se infiltró en Japón debido a su intenso intercambio comercial con Okinawa. Este aguardiente era muy parecido al que se elaboraba en Tailandia a base de arroz, llamado Rao Rong. Como es de suponer, junto con el aguardiente también llegó el material (arroz) y la técnica de producción, mismos que contribuyeron al nacimiento del awamori o shochu de Okinawa, es decir el shochu de la etapa inicial. Posteriormente la bebida llegó a la isla de Kyushu, una de las cuatro principales de Japón, situada en el oeste). ${ }^{3}$

El registro más antiguo de la palabra shochu en Japón está en el templo sintoísta Kouriyama Hachiman, en la ciudad de Oguchi, y data del año 1559. Es una inscripción tipo graffiti que hicieron dos carpinteros que trabajaban para la remodelación de este templo sintoísta, quejándose de que el encargado del templo nunca les ofreció el shochu. Existe poca información en Japón sobre esta técnica de destilación casi abandonada y sustituida por los modernos

3. Véase: http://www.japansake.or.jp/honkaku/about/all/history.html. 
complejos basados en destilación continua o de alambiques. ${ }^{4}$ Por ejemplo, no existen documentos en inglés sobre el aparato pero sí las obras de referencia en japonés donde se encuentra la descripción de la evolución de la máquina destiladora, los tipos y autenticidad de bebidas y sus materias primas. ${ }^{5}$ En la figura 2 podemos observar la evolución del aparato kabutogama chiki, según el libro Nihon no sake no rekishi (Historia de licores en Japón) escrito por el supervisor Keiichiro Sakaguchi y editado por Shinzaburo Kato (Editorial Kenseisya, pp. 534-537, sin fecha) según la entrevista realizada al señor Fukuda (2007, comunicación personal).

\section{Figura 2}

\section{Evolución del destilador kabutogama chiki}
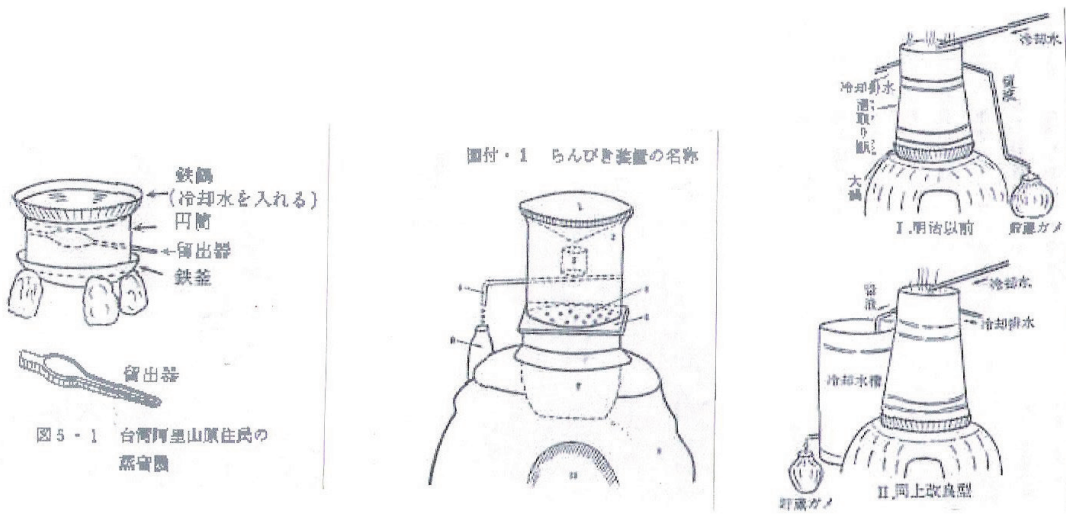

El destilador de influencia árabe desplazó a los aparatos primitivos. Paulatinamente, el destilador tipo kagutogama fue desapareciendo. Primero dio paso a diferentes versiones del mismo destilador conocidas en la isla Hachijo como "danbiki" hasta finales de la era Meiji (hasta 1912) (Noshiro, 1951).

4. Existen otros destiladores de este tipo pero como piezas de museo en (1) EmpresaTaen, Museo de Shochu (http://www.denen-shuzo.co.jp/museum.htm) y Empresa productora de licor Satsuma museo "Bodega Meiji" (http://www.satsuma.co.jp/s_satsuma/history03.html) y en el centro comunitario Chuo del pueblo Morozuka en la prefectura Miyazaki en exhibición con la explicación del método de destilación.

5. Dai san no sake (El tercer licor) del autor Seinosuke Kanma publicado en 1975 por la Editorial Asahi sonoraza y el otro es Shochu no hanashi (Cuentos de Sochu) editado en 1985 por la Editorial Gihodo (sin autor; Fukuda, 2007, comunicación personal). 
Sin embargo, cinco empresas o marcas de Shochu japonés situadas al oeste y al sur, con 218 productores (Kagoshima y Okinawa, encontradas en esta investigación) destilan todavía limitados volúmenes a partir del kabutogama chiki reconociendo en su etiqueta este signo distintivo tradicional, mas no se tienen estadísticas de producción. ${ }^{6}$

El destilador asiático que acabamos de referir, es decir el kabutogama chiki, es sorprendentemente muy parecido al que se usa para la producción de raicilla de El Tuito, en particular. En la fotografía 6 observamos el aparato destilador usado en la actualidad para el shochu, marca Nishikinada, de la prefectura de Kagoshima (http://www.pat.hi-ho.ne.jp/nanchan/nisikinada.html).
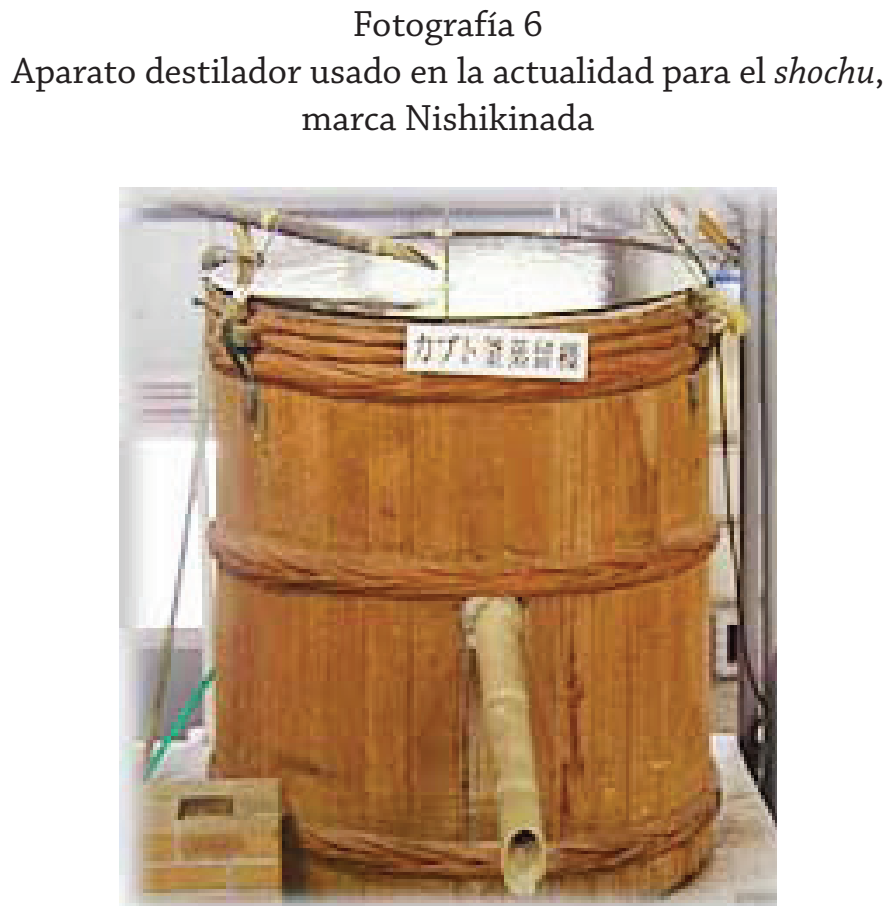

6. Productor Nishikinada (Kagoshima) (http://www.pat.hi-ho.ne.jp/nanchan/nisikinada.htmlhttps://secsvr.net/shochu-net.com/item.cgi?item_id=chintarari\&ctg_id=Shochu\&page=1); productor Ohishi (Kagoshima) (http://www.yoppawriter.com/tsukurite/index.html); productor Satsuma (Kagoshima), se usa este destilador para algunos productos limitados de su bodega Meiji; productor Kiyofuku (Kagoshima) es awamori (aguardiente especial de Okinawa), pero tienen algunos productos hechos con este destilador; Bodega Tonokura (Fukuoka). 
Esta es una prueba contundente de la influencia de los destiladores asiáticos en las costas de México para la producción de raicilla, por encima de los destiladores árabes. El destilador asiático (tal vez por su facilidad para construirse con materiales de la región costa: madera bonete para la torre de destilación, olla de cobre para el enfriador, penca de agave para conducir el destilado hasta la olla, etc.) fue adoptado en lugar del destilador tipo árabe, que requería de una mayor ingeniería, y se utilizó para destilar los fermentos de agave que en otros lugares se procesaban con alquitara.

Sin embargo, todavía es arriesgado considerar que los aparatos de destilación asiática sean la única influencia en la elaboración de destiladores jaliscienses, porque existe una gran variedad de aparatos a lo largo de Jalisco con particularidades diversas. Por eso, es más prudente privilegiar, para algunos casos, la pista de los ajustes y adaptaciones en los destiladores. Lo que sí resulta palpable es la gran influencia que tuvo Asia. Por otro lado, no hay que olvidar la teoría del modelo de vino de cocos y aguardiente, que sostiene que hubo una sola ruta para la propagación de la tradición de destilación tomando como punto de distribución la cuenca del río Ayuquila. Respecto a este tema conviene realizar algunas reflexiones históricas sobre la circulación del conocimiento por el mundo entero desde el siglo XVI, y considerar ciertas pistas que se abren a partir de esos análisis.

\section{La circulación de conocimientos en el siglo XVI}

En la ribera del río Guadalquivir, en una ciudad española que tiene algo de morisca, de gitana y de europea, acosta un navío llamado "El Victoria", que, aunque de apariencia antigua, es una embarcación de reciente factura que se erigió para conmemorar aquel 6 de septiembre de 1522 (Bénat-Tachot y Lavallé, 2005: 41) cuando un grupo escueto de 18 harapientos, "con pasos vacilantes" (Parry, 1991: 334) hizo un desembarco modesto pero ansiado en Sevilla. Eran los sobrevivientes de una nutrida expedición que había zarpado hacía tres años, en 1519, de San Lúcar, al mando de Fernando Magallanes bajo cuya responsabilidad se encontraban las cinco embarcaciones y los 250 hombres de que se componía la tripulación. Casi todos eran españoles, pero también había originarios de otras naciones europeas, y lo más sorprendente, había dos hombres malayos. Sólo un mes antes, en agosto de 1519, Hernán Cortés había iniciado la marcha hacia México-Tenochtitlan a partir de Veracruz. Es decir que cuando aún faltaba todo un continente por descubrir e incorporar 
a la dinámica europea, los objetos, los productos del extremo oriente, los hombres malayos cargados de conocimientos asiáticos ya vivían en Europa. Y más todavía, pues se aprestaban a llegar a Asia por una ruta completamente desconocida. La flota de Magallanes costeó las tierras brasileñas, bajó hasta la Patagonia y llegó a las aguas del Pacífico con dos embarcaciones menos. Sorteando el hambre y la enfermedad atravesaron el inmenso océano, donde permanecieron casi cuatro meses hasta llegar a las islas Marianas. La primera isla del archipiélago filipino que se le atravesó fue la de Guam. En toda esa región insular existía una red de comunicaciones marítimas avanzadas "donde los chinos dominaban la navegación comercial, aunque muchos otros pueblos participaban también en ellas” (Parry, 1991: 331). Intercambios, mestizajes, aculturaciones, transculturaciones... La comunicación y los vínculos asiáticos, desde principios del siglo XVI, incorporaron y fueron incorporados de manera directa al mundo europeo. Magallanes murió en la isla de Cebú, antes de iniciar el regreso a España. Juan Sebastián Elcano tomó el relevo y decidió el retorno por la vía del cabo de Buena Esperanza. Pero no tomó la ruta practicada comúnmente por los barcos portugueses, bogó siempre más al sur, en aguas oceánicas, porque quería evitar ser visto y combatido por los lusitanos. De modo que el regreso también fue un vía crucis, plagado de enfermedades, de hambre, de incertidumbres y de falta de comunicación. Por eso, un día después de haber desembarcado en Sevilla, los 18 sobrevivientes sintieron la imperiosa necesidad de hacer una visita al santuario de Santa María de la Victoria.

El dúo Fernando Magallanes-Sebastián Elcano realizó la primera vuelta al mundo, o como lo escribió Antonio Pigafetta de Vicenza en su diario, pues fue uno de los 18 sobrevivientes: "il Primo viaggio intorno al Mondo". Lejos de ser una mera anécdota, este acontecimiento (incluso de manera simbólica) marca una etapa de gran relevancia para comprender la historia de los mestizajes, de las transculturaciones y de las mundializaciones. Contrariamente a lo que se cree en nuestros días, ya han existido otras globalizaciones con las mismas características de fondo que la de la actualidad. La primera de ellas, bien documentada y analizada por el historiador Serge Gruzinski, es la de los siglos XVI-XVII, impulsada por los españoles y portugueses (Gruzinski, 2004). Las características de esta primera globalización, como la de ahora, eran la circulación alrededor del mundo de hombres, objetos y conocimientos; la tendencia a la uniformidad de prácticas y costumbres, de maneras de vestir y de actuar, de formas de producción, de sistemas de explotación... El "des- 
cubrimiento" de América por los europeos, la competencia por las especias asiáticas, la vuelta al mundo son algunos de los ingredientes que detonaron este proceso globalizante, del cual las grandes ciudades del planeta, México, Goa, Manila, Lisboa, Sevilla, Nápoles, constituían los eslabones de conexión entre el mundo y los lugares desde donde se distribuían los conocimientos planetarios. A su regreso del Caribe, Cristóbal Colón llevó a Europa nativos de las islas que llevaban consigo un lenguaje, costumbres y conocimientos nuevos para los europeos, y que se añadían al intercambio milenario que se tenía con los continentes africano y asiático. Pero este proceso de intercambios se aceleró en el siglo XVI y se hizo de manera directa entre Europa y Asia. La presencia de malayos en España en 1519 es un hecho revelador y muestra sólo la punta del iceberg sobre la presencia asiática en la península ibérica en esas fechas. Ese hecho también atestigua lo avanzado del proceso de mundialización.

Ante una realidad tan compleja en cuanto a la circulación de hombres, de objetos y de conocimientos hacia todas las direcciones del planeta (o "les quatre parties du monde" según la expresión de Serge Gruzinski) es muy arriesgado aceptar la tesis de Bruman, retomada por Patricia Colunga y Daniel Zizumbo (2006), sobre la ruta de ingreso del proceso de destilación a México. Basados en Fritz y Bruman (2000), ambos autores sugieren que, procedente de Filipinas, la tecnología asiática del destilado llegó a México por la vía de la costa de Jalisco y Colima:

The crucial event for the origin of Agave spirits was the introduction of the still in Colonial times (Fritzman y Bruman). Within the "Mezcal-Jocote" cultural area, this introduction derived originally from the Filipinos brought by the Spaniards to the coasts of Colima and Jalisco in the Manila Galleons in the late 16th century (Fritzman y Bruman). The Filipinos introduced the elaboration of fermented and distilled drinks from coconuts (Cocos nucifera L.) with their own technology, using a still unlike any in Europe and which could be built with local materials, very different from the Arabian still made of copper, used at the time by the Spaniards (Colunga y Zizumbo).

La introducción de bebidas destiladas y la producción de las mismas habría llegado, siguiendo a estos autores, de esa manera a finales del siglo XVI, con el "galeón de Manila" a la costa de Colima y Jalisco, desde donde se expandió hacia el distrito de Tequila, la región de Los Altos y luego a los estados de Michoacán, Guerrero y Oaxaca. Finalmente, esta invasión del destilado habría alcanzado el norte de México: Sonora, San Luis Potosí y Tamaulipas. 
The 400-year-old history of Tequila and the other Agave spirits has been brought forward from its beginnings in the 16th century when the indigenous population living in the foothills of the volcanoes of Colima first submitted the fermented Agave beverage to the Filipino still. Its subsequent diffusion in the 17 th and 18th centuries, according to this author, was directed north, towards the district of Tequila and the region of "Los Altos" in the present-day state of Jalisco; to the south in the modern states of Michoacan, Guerrero and Oaxaca, after which it the spread to the mining centres of the north: Sonora, San Luis Potosi and Tamaulipas (Colunga y Zizumbo).

Esta visión es muy reducida y desdeña la dimensión, la profundidad y las posibilidades históricas de la circulación del conocimiento alrededor del planeta desde las primeras décadas del siglo XVI. Después de haber revisado a grandes rasgos el contexto histórico internacional del siglo del Renacimiento, sólo podemos aceptar que las posibilidades de que los conocimientos sobre la destilación asiática ingresaran a México incluso por Veracruz, son muy altas. Por otro lado, hay que aceptar que el método de destilación asiático no solamente se conocía en Filipinas, pues durante siglos había sido utilizado en Corea y Japón, como ya se dijo. En fin, no hay que olvidar que en una misma región de la costa de Jalisco, en lugares tan cercanos como San Sebastián y El Tuito, conviven actualmente dos métodos de destilación. En efecto, hemos podido constatar que el destilador de San Sebastián es un aparato híbrido con características del alambique árabe, con serpentina. Pero el destilador de El Tuito aún guarda similitudes sorprendentes con el asiático. Esto significa que los conocimientos de destilación llegaron por diferentes vías a diversos puntos del territorio mexicano, de manera simultánea. Falta echar a andar esta investigación y probarlo con evidencias. En todo caso, retomar este punto de vista dentro de un contexto histórico globalizante como lo es el siglo XVI, es una invitación a multiplicar las hipótesis y las problemáticas explicativas, salir de los modelos reduccionistas con el ánimo de avanzar en un campo de estudio todavía en ciernes. Retomar el estudio de los destilados en México considerando una perspectiva histórica de profundidad (ir más allá de la contextualización para que la Historia sirva de explicación) con nuevas hipótesis y nuevos métodos, desde diferentes perspectivas científicas, es decir con un espíritu interdisciplinario, es apostarle al avance del conocimiento científico y a la tendencia hacia su completitud. Esa es la propuesta. Llevarla a cabo sería comprender la amplitud del significado que encierra una embarcación renacentista que acosta en las orillas del río Guadalquivir, cerca del barrio de Triana. 


\section{Conclusiones}

Para cerrar esta invitación a la discusión y al debate es necesario recorrer otra vez, brevemente, el texto pero de manera inversa. Es decir, situarnos en ese siglo XVI descrito en la tercera parte de este artículo, cuando la circulación de conocimientos, de objetos y de hombres alrededor del mundo era una realidad. En ese siglo, los dos procesos de destilación (el árabe y el asiático) llegaron a México (por diversas rutas, probablemente) y fueron utilizados a lo largo de la costa del Pacífico para obtener aguardiente: de coco, en Colima, (Romero de Solís, 2008: 227-228) y de agave, en la costa de la Nueva Galicia (Arregui, 1980). Las fuentes históricas no muestran la presencia de destilados en el occidente mexicano para el siglo XVI. Sin embargo, para el siglo XVII hay testimonios irrefutables sobre la elaboración y utilización de aguardientes. ¿Por qué tal silencio para el siglo XVI?

Por el contrario, no faltan testimonios sobre la elaboración y consumo de destilados en Filipinas desde antes de la llegada de los españoles. Es otro indicio que muestra que si los españoles hubieran encontrado destilados prehispánicos en México no habrían dejado de escribirlo, no habría pasado inadvertido. Antonio de Morga escribió sin complejos que en la provincia de Cagayán:

\footnotetext{
[...] sacada la tuba de la palma, la destilan por alambiques en sus hornillos e instrumentos, con más o menos fuerza, y queda hecha aguardiente. Y esto se bebe en todas las islas [Filipinas]... los naturales destas islas en sus juntas, casamientos y fiestas, todo es beber deste vino, días y noches sin cesar, por su rueda cantando unos y otros bebiendo, de que muy ordinario viene a emborracharse, sin que entre ellos este vicio sea deshonra ni infamia (Morga, 2006: 225).
}

¿Por qué habría que pensar que en la más grande elocuencia y prolijidad de un Motolinía, o un Bernal Díaz del Castillo, o un Bernardino de Sahagún, se habría de ocultar la existencia de los destilados en México? Tal vez porque no los había.

Por otro lado, hay que resaltar el tipo de destilador asiático. Por lo pronto no se han encontrado descripciones precisas de este aparato, pero poseemos ilustraciones claras del destilador kabutogama chiki. Se trata de un instrumento idéntico al que utilizan actualmente algunos raicilleros de la costa de Jalisco. No puede haber prueba más contundente para reconocer la influencia asiática en el proceso de destilación de estos aguardientes desde tiempos aún no determinados hasta nuestros días. 
No podemos poner el punto final a este texto sin insistir en que los agaves eran utilizados de manera intensa en la época prehispánica como alimento, bebida y fibras. Las cinco especies de agave a las que se hizo referencia en este texto fueron dominadas y cultivadas siguiendo una herencia tradicional indígena. Sin embargo, en la actualidad los usos del agave y los procesos de destilación conocen una transición de lo artesanal a lo industrial, lo que puede provocar graves riesgos en términos de biodiversidad y de sustentabilidad del medio ambiente. Además, los productos obtenidos de manera industrial (como los destilados) disminuyen su calidad en comparación con los artesanales.

Nuevas líneas de investigación sobre la influencia de la destilación asiática en los mezcales de México podrán realizarse si se promueven estudios de campo en las tabernas artesanales, donde actualmente se pueden encontrar aparatos destiladores de raicilla tan parecidos al kabutogama chiki de Japón para producir el shochu. miv

\section{Bibliografia}

Arregui, Domingo Lázaro de, Descripción de la Nueva Galicia, Uned, Guadalajara, 1980 [1621].

Bénat-Tachot Louise y Bernard Lavallé, L'Amérique de Charles Quint, Presses Universitaires de Bordeaux, Burdeos, 2005.

Chaunu, Pierre, Conquête et exploitation des nouveaux mondes, PUF, París, 1969.

Colunga-García Marín P. y D. Zizumbo-Villarreal, "Tequila and other agave spirits from west-central Mexico: current germplasm diversity, conservation and origin", Journal of Biodiversity and Conservation, 2006, pp. 1-15. Disponible en: http://10.1007/s10531-006-9031-z. Issue: Online First. Fecha de consulta: 29 de octubre de 2008.

Consejo Mexicano Promotor de la Raicilla, 2000. Del archivo de la Organización no gubernamental-Asociación Civil propietaria de la marca colectiva Raicilla, reconocido por el Instituto Mexicano de la Propiedad Intelectual de México. Documento interno fechado el 10 de julio de 2000.

Dachary, Alfredo César, "Rutas, culturas y cotidianidad en el mundo rural". Ponencia presentada en el Seminario sobre Turismo Rural y su Contribución a la Creación de Empleo y la Conservación del Patrimonio, Asunción, Paraguay, 12 y 13 de mayo de 2003, pp. 16. Disponible en: http://infoagro.net/shared/ docs/a5/Aliartesanales2.pdf. Fecha de consulta: 22 de enero de 2009. 
Eguiarte, Luis, "Diversidad genética en dos especies mezcaleras", Informe final del proyecto V038. Proyecto a la Comisión Nacional de Uso y Conservación de la Biodiversidad, 2003. Disponible en: http://www.conabio.gob. $\mathrm{mx} /$ institucion/proyectos/resultados/InfV038.pdf. Fecha de consulta: 29 de octubre de 2008.

Fournier, Dominique, "Ferments de cultures", en Bessis, Sophie (dir.) Mille et une bouches. Cuisines et identités culturelles, Autrement, París, 1995. Disponible en: http://www.lemangeur-ocha.com/auteurs/habitudes-alimentaires/auteur/697/biblio/. Fecha de consulta: 29 de octubre de 2008.

Furst Peter y Henry J. Bruman, Alcohol in Ancient México, University of Utah Press, EU, 2000.

Gruzinski, Serge, Les quatre parties du monde. Histoire d'une mondialisation, La Martinière, Turín, 2004.

Hernández V. Gerardo, B. Miguel Cházaro y E. Flores-Berrios, "Inventario, distribución y hábitat del género Agave en Jalisco", en Agaves del occidente de México (serie Fronteras de Biodiversidad), 2007, núm. 221, pp. 6-12.

Illsley, G. C., "Los mezcales como productos regionales tradicionales". Ponencia en el CD Memorias del encuentro Los mezcales tradicionales de los pueblos de México: herencia cultural y biodiversidad, Ciudad de México, del 15 al 20 de agosto, 2005.

Macías M., Alejandro y A. G. Valenzuela-Zapata, "El campo económico del tequila en tiempos de globalización”, en Valenzuela-Zapata, A. G. y Gerritsen, P. R. W. Memorias. Tequila: ambiente, cultura y sociedad. Primer Seminario Internacional del Tequila SIT 2007, $1^{\circ}$ de diciembre de 2007, Guadalajara, Jalisco.

Morga, Antonio de, Sucesos de las Islas Filipinas, FCE, México, 2007 [1607].

Motolinía, Fray Toribio de, Historia de los indios de la Nueva España, Porrúa, México, 2001 [1541].

Noshiro, Kinichi, "Presente y pasado del Kyuuma Shochu de la prefectura de Kumamoto", Revista de la Asociación Japonesa de Productores de Licores, vol. 46, núm. 8, 1951, pp. 285-286.

Parry, John H., El descubrimiento del mar, Conaculta-Grijalbo, México, 1991.

Romero de Solís, José Miguel, "El conquistador Francisco Cortés. Reivindicación de un cobarde", Pretextos, Textos y Contextos, núm. 10, AHMC, Colima, 1996. 
- Clérigos, encomenderos, mercaderes y arrieros en Colima de la Nueva España (1523-1600), Morelia, Michoacán, AHMc-Universidad de Colima-El Colegio de Michoacán, 2008.

Standage, Tom, A history of World in 6 glasses. Nueva York, Walker and Company, 2005.

Valenzuela, Z. A. G., "Rutas alternativas aprovechando la agrobiodiversidad de agaves, Tequila, mezcal y pulque en Jalisco". Conferencia en las Jornadas de actualización de turismo del 9 al 11, abril, 2008. Centro Universitario del Sur. Zapotlán el Grande Jalisco, Universidad de Guadalajara, 2007.

Archivos, entrevistas y sitios de Internet

AGI: Archivo General de Indias. En Sevilla, España.

Fukuda, 2007. Comunicación personal. Entrevista de Michiko Mizoguchi al señor Fukuda, del National Research Institute of Brewing, en Tokio, Japón, sobre las fuentes bibliográficas del aparato destilador kabutogama shiki para la producción artesanal de shochu. Enero, 18, 2008.

Justo, Rafaela, 2007. Comunicación personal. Entrevista de Ana G. Valenzuela Z. a la Presidenta de Pueblos Indígenas de Manantlán, la licenciada Rafaela Justo, sobre la tradición de la bebida "lechuguilla" del municipio de Cuautitlán, Jalisco, a partir de Agave inaequidens, en el centro de Ecocuexco, Tlajomulco de Zúñiga, el 10 de agosto de 2008.

Sitios web

http://www.denen-shuzo.co.jp/museum.htm. www.japansake.or.jp/honkaku/about/all/history.html.

www.jcmiras.net/jcm2/p16.htm\#more-16.

www.rae.es

www.satsuma.co.jp/s_satsuma/history03.html.

www.shochu.com.

\section{Agradecimientos}

A los fibreros, pulqueros, mezcaleros y raicilleros de Autlán, El Tuito, Mascota, San Sebastián, Zapotitlán de Vadillo, Zacoalco y Tapalpa por su información. A los señores Fukuda y Utsunomiya, del National Research Institute of Brewing, por permitirnos bibliografía y concedernos entrevistas. 